\title{
Near-infrared spectroscopy of a nitrogen-loud quasar SDSS J1707+6443
}

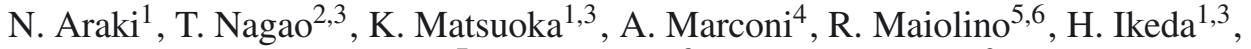 \\ T. Hashimoto ${ }^{7}$, Y. Taniguchi ${ }^{8}$, and T. Murayama ${ }^{9}$
}

\author{
${ }^{1}$ Graduate School of Science and Engineering, Ehime University, 2-5 Bunkyo-cho, 790-8577 Matsuyama, Japan \\ 2 The Hakubi Center for Advanced Research, Kyoto University, Yoshida-Ushinomiya-cho, Sakyo-ku, 606-8302 Kyoto, Japan \\ 3 Department of Astronomy, Graduate School of Science, Kyoto University, Kitashirakawa-Oiwake-cho, Sakyo-ku, 606-8502 Kyoto, \\ Japan \\ 4 Dipartimento di Fisica e Astronomia, Università degli Studi di Firenze, Largo E. Fermi 2, 50125 Firenze, Italy \\ 5 INAF - Osservatorio Astrofisico di Roma, Via di Frascati 33, 00040 Monte Porzio Catone, Italy \\ ${ }^{6}$ Cavendish Laboratory, Univerisy of Cambridge, 19 J. J. Thomson Ave., Cambridge CB3 0HE, UK \\ 7 Optical and Infrared Astronomy Division, National Astronomical Observatory of Japan, 2-21-1 Osawa, 181-8588 Mitaka, Japan \\ 8 Research Center for Space and Cosmic Evolution, Ehime University, 2-5 Bunkyo-cho, 790-8577 Matsuyama, Japan \\ 9 Astronomical Institute, Graduate School of Science, Tohoku University, Aramaki, Aoba, 980-8578 Sendai, Japan
}

Received 18 November 2011 / Accepted 21 May 2012

\section{ABSTRACT}

\begin{abstract}
We present near-infrared spectroscopy of the $z \simeq 3.2$ quasar SDSS J1707+6443, obtained with MOIRCS on the Subaru Telescope. This quasar is classified as a "nitrogen-loud" quasar because of the fairly strong $\mathrm{N} \mathrm{III]} \mathrm{and} \mathrm{N} \mathrm{IV]} \mathrm{semi-forbidden} \mathrm{emission} \mathrm{lines} \mathrm{from}$ the broad-line region (BLR) observed in its rest-frame UV spectrum. However, our rest-frame optical spectrum from MOIRCS shows strong [O III] emission from the narrow-line region (NLR), suggesting that, at variance with the BLR, NLR gas is not metal-rich. To reconcile these contradictory results, there may be two alternative possibilities: (1) the strong nitrogen lines from the BLR are simply caused by a very high relative abundance of nitrogen and not by a very high BLR metallicity, or (2) the BLR metallicity is not representative of the metallicity of the host galaxy, which is better traced by the NLR. In either case, the strong broad nitrogen lines in the UV spectrum are ot indication of a chemically enriched host galaxy. We estimated the black hole mass and Eddington ratio of this quasar from the velocity width of both $\mathrm{C}$ IV and $\mathrm{H} \beta$, which results in $\log \left(M_{\mathrm{BH}} / M_{\odot}\right)=9.50$ and $\log \left(L_{\mathrm{bol}} / L_{\mathrm{Edd}}\right)=-0.34$. The relatively high Eddington ratio is consistent with our earlier result that strong nitrogen emission from BLRs is associated with high Eddington ratios. Finally, we detected significant [Ne III] emission from the NLR, implying a quite high gas density of $n_{\mathrm{e}} \sim 10^{6} \mathrm{~cm}^{-3}$ and suggesting a strong coupling between quasar activity and dense interstellar clouds in the host galaxy.
\end{abstract}

Key words. galaxies: active - galaxies: nuclei - quasars: emission lines - quasars: individual: SDSS J1707+6443

\section{Introduction}

The chemical composition of galaxies is a powerful tool to distinguish various evolutionary scenarios because it is the result of past star-formation activity and is also affected by gas inflows and outflows. The chemical properties of active galactic nuclei (AGN) are particularly interesting, because the huge luminosities of AGN enable us to accurately measure spectroscopic features of quasars even at high redshifts and thus to explore the chemical properties in the early universe. Another interesting aspect of assessing the chemical composition of AGNs is that it provides clues on the co-evolution between galaxies and their supermassive black holes (SMBHs), which has been inferred by the tight correlation between the mass of SMBHs $\left(M_{\mathrm{BH}}\right)$ and their host spheroidal observed in the local Universe (e.g., Marconi \& Hunt 2003; Ferrarese \& Merritt 2000; Gebhardt et al. 2000). Matsuoka et al. (2011) reported a tight relationship between the metallicity in broad-line regions (BLRs) and $M_{\mathrm{BH}}$ at $z \sim 2-3$, suggesting that the evolution of SMBHs is associated with the cumulative star formation in the host galaxies (see also Warner et al. 2004; Nagao et al. 2006b).

A widely adopted method of estimating the BLR metallicity $\left(Z_{\mathrm{BLR}}\right)$ exploits the flux of nitrogen emission lines such as
$\mathrm{N} v \lambda 1240$. Nitrogen is a secondary element and thus its relative abundance is proportional to the metallicity, $[\mathrm{N} / \mathrm{O}] \propto[\mathrm{O} / \mathrm{H}]$ or, equivalently, $[\mathrm{N} / \mathrm{H}] \propto[\mathrm{O} / \mathrm{H}]^{2}$ (see, e.g., Hamann \& Ferland 1992). Based on this method, it has been found that the BLR in most quasars show super-solar metallicities (e.g., Nagao et al. 2006b), even for quasars at $z \gtrsim 6$ (Jiang et al. 2007; Juarez et al. 2009; Mortlock et al. 2011). The BLR metallicity is up to $Z_{\mathrm{BLR}} \sim 10 Z_{\odot}$ in the most extreme cases although these high metallicities are hard to understand with ordinary galaxy chemical evolution models (e.g., Hamann \& Ferland 1993). More interestingly, some quasars show extremely strong emission in $\mathrm{N} v$ and in other nitrogen lines (especially $\mathrm{N}$ IV $] \lambda 1486$ and $\mathrm{N}$ III] $\lambda 1750$ semi-forbidden lines). The estimated $Z_{\mathrm{BLR}}$ reaches $\sim 15 Z_{\odot}$, or higher, and these quasars are classified as " $\mathrm{N}$-loud quasars" (e.g., Baldwin et al. 2003; Bentz \& Osmer 2004; Bentz et al. 2004). Here the definition of a N-loud quasar is that of Jiang et al. (2008); i.e., type 1 quasars with strong nitrogen emission of $E W_{\text {rest }}(\mathrm{N}$ IV $\left.] \lambda 1486\right)>3 \AA$ or $E W_{\text {rest }}(\mathrm{N}$ III $\left.] \lambda 1750\right)>$ $3 \AA$ (see Sect. 2). Note that the measurement of the $\mathrm{N} v \lambda 1240$ flux is sometimes uncertain because of the heavy blending with the Ly $\alpha$ emission (see, e.g., Nagao et al. 2006b); however, the $\mathrm{N}$-loudness of these quasars is convincing because the wellisolated $\mathrm{N}$ Iv] $\lambda 1486$ and $\mathrm{N}$ III] $\lambda 1750$ are used for defining the 
N-loud quasar population. Standard chemical evolution models cannot predict these extremely high metallicities at any epoch (e.g., Ballero et al. 2008), therefore these N-loud quasars is a great challenge for galaxy evolutionary models.

Jiang et al. (2008) pointed out that N-loud quasars may not have such high $Z_{\mathrm{BLR}}$, but simply have an unusually high relative abundance of nitrogen in the BLR, mainly because the emission-line spectrum of N-loud quasars is not significantly different from that of typical quasars except for the nitrogen lines. This idea questions the use of the $\mathrm{N} v$ emission for $Z_{\mathrm{BLR}}$ measurements in quasars. It is therefore very important to verify observationally if $\mathrm{N}$-loud quasars have either high $Z_{\mathrm{BLR}}$ or just high nitrogen relative abundances. More recently, Matsuoka et al. (2011) reported that the strength of nitrogen lines is correlated with the quasar Eddington ratio $\left(L_{\mathrm{bol}} / L_{\mathrm{Edd}}\right)$ and discussed the possibility that quasars with strong nitrogen emission are in a special phase of the evolutionary history of their host galaxies. These studies outline the importance of understanding the nature of N-loud quasars, especially in terms of their chemical evolution. Therefore it is important to assess the metallicity of BLRs and host galaxies of $\mathrm{N}$-loud quasars with a method that does not rely on rest-frame UV broad nitrogen lines.

In this paper, we focus on the emission of the narrow-line region (NLR) in N-loud quasars. Since strong nitrogen lines from their BLRs suggest a high $Z_{\mathrm{BLR}}$, we examined the properties of the NLR to independently investigate the metallicity in the N-loud quasar population. While the BLR is located in very compact, subpc-scale regions around SMBHs, NLR gas clouds are distributed on kpc scales, i.e. on the scales of the host galaxies, which enabled us to study the possible relationships between the properties of N-loud quasars and their host galaxies. Throughout this paper, we adopt a cosmology with $H_{0}=70 \mathrm{~km} \mathrm{~s}^{-1} \mathrm{Mpc}^{-1}, \Omega_{\mathrm{m}}=0.3$, and $\Omega_{\Lambda}=0.7$.

\section{Sample selection, observation, and the data reduction}

To investigate NLR properties of N-loud quasars in detail, we focused on SDSS J1707+6443 at $z=3.163$ from the N-loud quasar catalog of Jiang et al. (2008). This catalog contains 293 N-loud quasars selected from the Fifth Data Release quasar catalog (Schneider et al. 2007) of the Sloan Digital Sky Survey (SDSS; York et al. 2000). The N-loud quasars in the catalog of Jiang et al. (2008) were selected according to the following criteria: (i) $i^{\prime}<20.1$; (ii) $1.7<z<4.0$; and (iii) $E W_{\text {rest }}(\mathrm{N}$ IV $\left.] \lambda 1486\right)>$ $3 \AA$ or $\left.E W_{\text {rest }}\left(\mathrm{N}_{\text {III }}\right] \lambda 1750\right)>3 \AA$. Among the N-loud quasars listed in the catalog of Jiang et al. (2008), we focused on SDSS J1707+6443 because it is relatively bright and its redshift is adequate for detecting redshifted NLR lines without suffering from the deep atmospheric absorption (Fig. 1). In Fig. 2, we show the frequency distributions of $E W_{\text {rest }}(\mathrm{N}$ IV $\left.] \lambda 1486\right)$ and $\left.E W_{\text {rest }}(\mathrm{N} \mathrm{III]}] 21750\right)$, and also where SDSS J1707+6443 is located in these frequency distributions (see also Table 1). The averages and standard deviations of $E W_{\text {rest }}(\mathrm{N}$ IV $\left.] \lambda 1486\right)$ distributions are $4.6 \AA \pm 1.7 \AA$, with minimum and maximum values in the parent sample of $1.2 \AA$ and $8.9 \AA$. The averages and standard deviations of $E W_{\text {rest }}(\mathrm{N}$ III] $\lambda 1750)$ are $5.6 \AA \pm 2.6 \AA$, with minimum and maximum values of $2.5 \AA$ and $27.1 \AA$. Figure 2 does not suggest that SDSS J1707+6443 is located at the lowest end in the distribution of $E W_{\text {rest }}(\mathrm{N}$ III] $\lambda 1750)$, because some of $\mathrm{N}$-loud quasars do not always show detectable emission lines of $\mathrm{N}$ Iv] $\lambda 1486$ and $\mathrm{N}$ III] $\lambda 1750$. More specifically, only $50 \mathrm{~N}$-loud quasars out of 293 show detectable $\mathrm{N}$ IV] $\lambda 1486$ emission

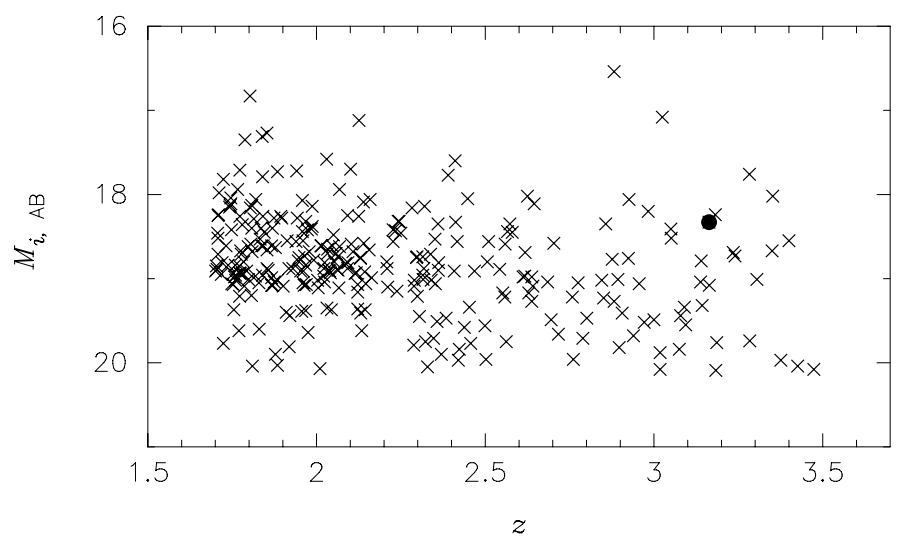

Fig. 1. Absolute $i^{\prime}$-band magnitudes of $293 \mathrm{~N}$-loud quasars as a function of redshift. Filled circle and crosses denote SDSS J1707+6443 and the other N-loud quasars, respectively. Data are taken from Jiang et al. (2008).
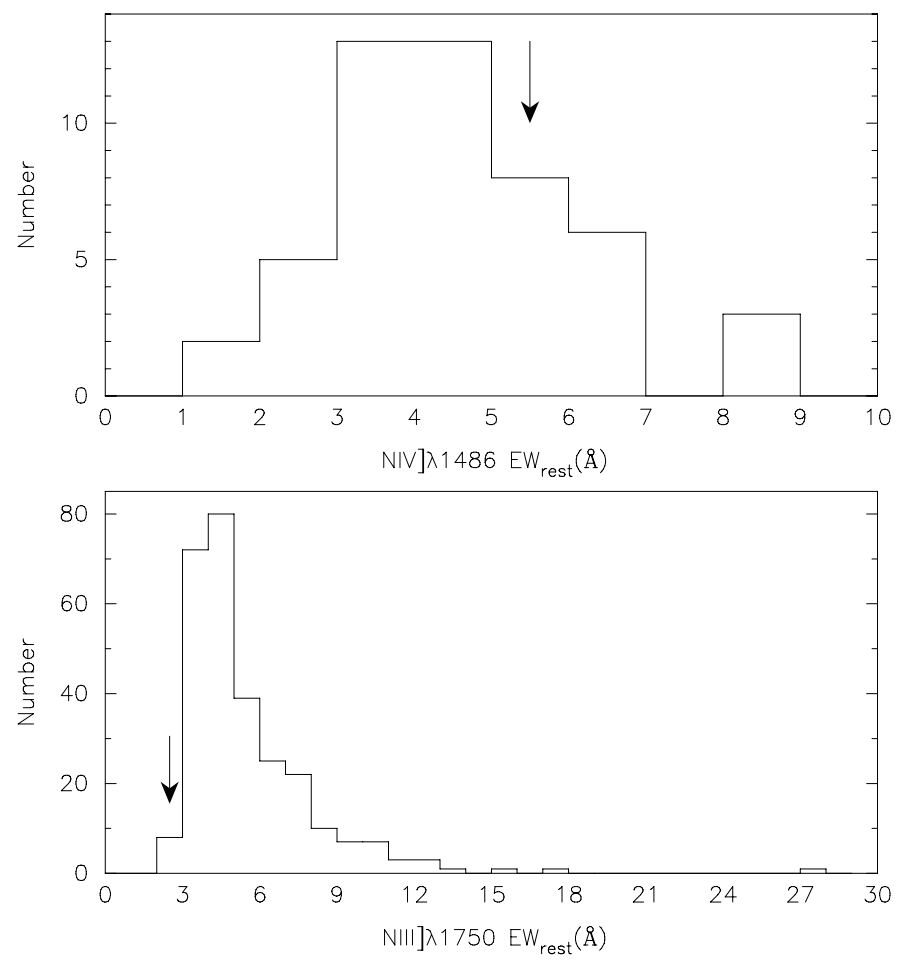

Fig. 2. Histograms of $E W_{\text {rest }}$ of $\mathrm{N}$ IV] $\lambda 1486$ (upper panel) and $\mathrm{N}$ III] $\lambda 1750$ (lower panel). The arrows denote the $E W$ rest values of SDSS J1707+6443. Data are taken from Jiang et al. (2008).

while 280 out of 293 show detectable $\mathrm{N}$ III] $\lambda 1750$ emission (Jiang et al. 2008). Although SDSS J1707+6443 has a high $\mathrm{N}$ IV] $\lambda 1486 / \mathrm{N}$ III] $\lambda 1750$ ratio with respect to typical $\mathrm{N}$-loud quasars, we do not discuss this question in this paper since it is beyond the scope of this work. The SDSS spectrum and the basic observational properties of SDSS J1707+6443 are shown in Fig. 3 and Table 1.

To assess the NLR properties of type 1 quasars, it is necessary to investigate rest-frame optical spectra and consequently, near-infrared spectroscopic data are required for this target. Therefore we observed SDSS J1707+6443 with MOIRCS (Ichikawa et al. 2006; Suzuki et al. 2008), the near-infrared spectrograph at the Subaru Telescope, on 30 May 2009. We used the HK grism, which yielded a wavelength coverage of 
Table 1. Observational properties of SDSS J1707+6443.

\begin{tabular}{lcccccccc}
\hline \hline \multirow{2}{*}{ Name } & \multirow{2}{*}{$i_{\mathrm{AB}}^{\prime}(\mathrm{mag})$} & $i^{\prime}{ }_{\text {abs,AB }}(\mathrm{mag})^{a}$ & $H_{\text {Vega }}(\mathrm{mag})^{b}$ & \multicolumn{3}{c}{$E W_{\text {rest }}(\AA)^{c}$} \\
& & & & & $\mathrm{~N}$ IV $]$ & $\left.\mathrm{N}_{\text {III }}\right]$ & $\mathrm{C}$ IV & $\mathrm{C}_{\text {III }}$ \\
\hline SDSS J1707+6443 & 3.163 & 18.33 & -28.06 & 16.15 & 5.1 & 2.5 & 40.8 & 15.3 \\
\hline
\end{tabular}

Notes. ${ }^{(a)} i^{\prime}$ band absolute magnitude given by Schneider et al. (2007); ${ }^{(b)}$ 2MASS magnitude given by Schneider et al. (2007); ${ }^{(c)}$ rest-frame equivalent widths of some BLR lines given by Jiang et al. (2008).

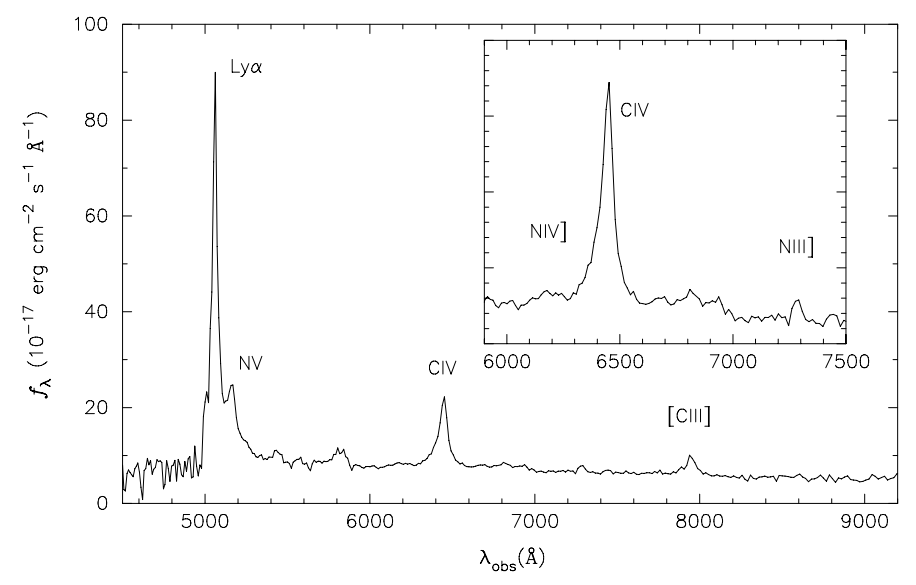

Fig. 3. Optical spectrum of SDSS J1707+6443 obtained from the SDSS database. Line IDs are given for strong emission lines. The inset shows spectral features around the $\mathrm{C}$ Iv emission, where $\mathrm{N}$ iv] $\lambda 1486$ and $\mathrm{N}$ III] $\lambda 1750$ lines are seen.

$1.4 \mu \mathrm{m} \lesssim \lambda_{\text {obs }} \lesssim 2.3 \mu \mathrm{m}$. By using the $0.6^{\prime \prime}$-wide slit, the resulting spectral resolution was $R \sim 570$, which was measured through the widths of the observed $\mathrm{OH}$ airglow emission. The total integration time was $1800 \mathrm{~s}$, consisting of six $300 \mathrm{~s}$ independent exposures. We also observed HIP 86687 (A2 star with $H_{\text {Vega }}=8.9$ and an assumed effective temperature of $8810 \mathrm{~K}$ ) for the flux calibration and the telluric absorption correction. During the observation, the typical seeing size was $0.8^{\prime \prime}$ in the optical.

Standard data processing was performed using the available $\mathrm{IRAF}^{1}$ routines. Frames were flat-fielded using domeflat images, and sky emission subtraction was performed by subtracting pairs of subsequent frames with the target at different positions along the slit. Cosmic-ray events were then removed by using the lineclean task. We extracted the one-dimensional spectrum of the target using the apall task, with an aperture size of \pm 5 pixels $\left(1.17^{\prime \prime}\right)$ from the emission center and, in this process, sky residuals were removed. Wavelength calibration was performed using $\mathrm{OH}$ sky lines. Finally, flux calibration and telluric absorption correction were carried out using the observed spectra of HIP 86687. The final processed spectrum was obtained by combining all single exposure frames.

To check the consistency of the flux calibration between the SDSS data and our MOIRCS data, we extrapolated the UV continuum emission toward the longer wavelength and compared the flux density at $\lambda_{\text {rest }}=5100 \AA$ between the extrapolated spectrum and our MOIRCS spectrum. The adopted spectral index is derived by fitting the SDSS spectrum at the wavelength regions where strong emission-line features are not present $\left(\lambda_{\text {rest }} \sim\right.$ $1350 \AA, 1450 \AA, 1670 \AA$, and $1970 \AA$ ). Here we assume a

\footnotetext{
${ }^{1}$ IRAF is distributed by the National Optical Astronomy Observatory, which is operated by the Association of Universities for Research in Astronomy (AURA) under cooperative agreement with the National Science Foundation.
}

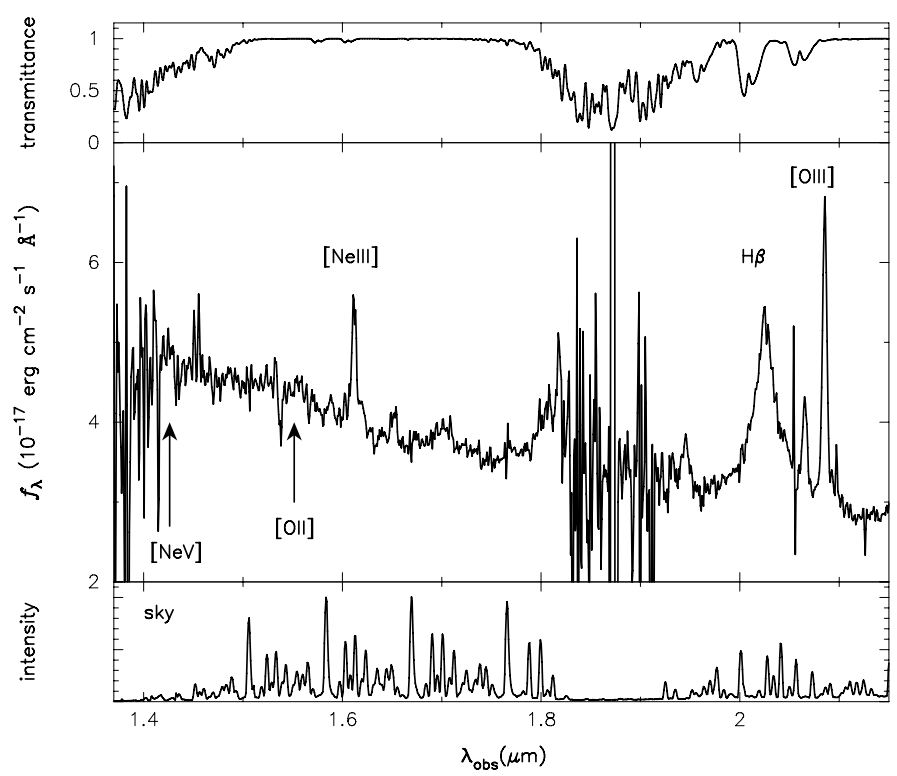

Fig. 4. Near-infrared spectrum of SDSS J1707+6443 obtained in our MOIRCS run (middle panel), with the Mauna Kea atmospheric transmission (upper panel) and the typical sky spectrum obtained during our MOIRCS run (lower panel). Important emission lines are labeled in the middle panel.

constant spectral index at $\lambda_{\text {rest }}<4000 \AA$ (see Vanden Berk et al. 2001). The discrepancy in the continuum flux is $\sim 10 \%$, and therefore we conclude that the flux calibration is consistent between the SDSS spectrum and our MOIRCS spectrum.

\section{Result}

The final processed spectrum of SDSS J1707+6443 is shown in Fig. 4, with the Mauna Kea atmospheric transmission ${ }^{2}$ and a typical sky spectrum obtained during our MOIRCS observing run. A prominent broad $\mathrm{H} \beta$ emission is detected at $\lambda_{\mathrm{obs}} \sim 2.0 \mu \mathrm{m}$, a typical spectral feature from quasar BLRs. In addition to the broad $\mathrm{H} \beta$ emission, some narrow emission lines from the NLR are also detected, like the [O III] doublet in the $K$-band, a feature common in several high-z quasars (e.g., McIntosh et al. 1999; Netzer et al. 2004; Marziani et al. 2009; Greene et al. 2010). Interestingly, we also detected the quite rare [Ne III] $\lambda 3869$ emission line but not the $[\mathrm{Ne} \mathrm{v}] \lambda 3426$ and [O II] $\lambda 3727$ lines.

To measure the emission-line fluxes and velocity widths, we fit the spectral features by using the specfit routine (Kriss 1994). Here we adopted a single Gaussian profile for forbidden narrow lines and a double Gaussian profile for $\mathrm{H} \beta$ line. The measured emission-line properties are summarized in Table 2, where the presented quantities are based on the fitting models, not on the actual data themselves. In Fig. 5, we show

${ }^{2}$ Data obtained from the UKIRT web site. 
Table 2. Measured spectral features.

\begin{tabular}{lccccc}
\hline \hline Line ID & $\begin{array}{c}\text { Center wavelength } \\
{[\AA]}\end{array}$ & $\begin{array}{c}E W_{\text {rest }} \\
{[\AA]}\end{array}$ & $\begin{array}{c}\text { Line flux } \\
{\left[10^{-17} \mathrm{erg} \mathrm{s}^{-1} \mathrm{~cm}^{-2}\right]}\end{array}$ & $\begin{array}{c}\mathrm{FWHM}^{a} \\
{[\AA]}\end{array}$ & $\begin{array}{c}\mathrm{FWHM}^{b} \\
{\left[\mathrm{~km} \mathrm{~s}^{-1}\right]}\end{array}$ \\
\hline$[\mathrm{Ne}$ v $] \lambda 3426$ & - & - & $<12.3^{c}$ & - & - \\
{$[\mathrm{O}$ II] $] \lambda 3727$} & - & - & $<10.0^{c}$ & - & - \\
{$[\mathrm{Ne}$ III] $] \lambda 3869$} & 16117.5 & $4.9 \pm 0.2$ & $83.3 \pm 3.7$ & $54.5 \pm 6.5$ & $866 \pm 103$ \\
$\mathrm{H} \beta_{\text {narrow }}$ & 20245.7 & $4.1 \pm 0.2$ & $51.8 \pm 2.3$ & $55.3 \pm 8.4$ & $627 \pm 95$ \\
$\mathrm{H} \beta_{\text {broad }}$ & 20267.1 & $49.3 \pm 0.5$ & $628.6 \pm 5.9$ & $379.9 \pm 67.8$ & $5595 \pm 999$ \\
{$[\mathrm{O}$ III $] \lambda 4959$} & 20653.8 & $5.8 \pm 0.1$ & $72.5 \pm 1.7$ & $56.4 \pm 8.6$ & $627 \pm 96$ \\
{$[\mathrm{O}$ III $] \lambda 5007$} & 20853.7 & $17.7 \pm 0.1$ & $217.9 \pm 1.7$ & $57.0 \pm 8.7$ & $627 \pm 96$ \\
\hline
\end{tabular}

Notes. ${ }^{(a)}$ Measured emission-line width before the correction for the instrumental broadening; ${ }^{(b)}$ emission-line velocity width after the correction for the instrumental broadening; ${ }^{(c)}$ for the undetected lines, $3 \sigma$ upper-limit fluxes are given.

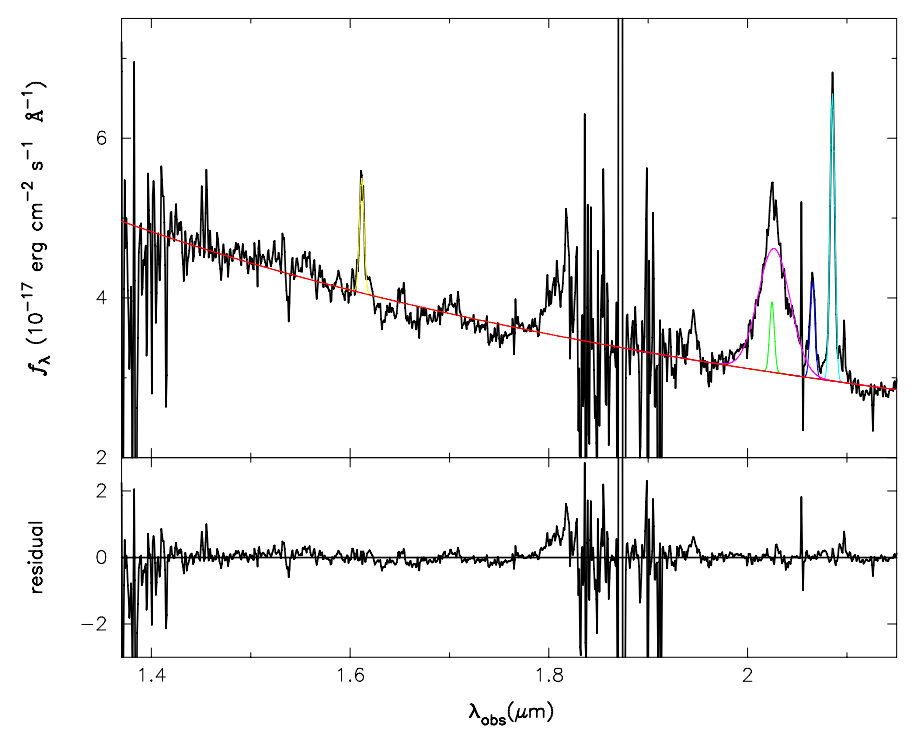

Fig. 5. Fitting result (upper panel) and the residual (lower panel) for the processed MOIRCS spectrum. See the main text for details of the fitting procedure.

fitting results and residuals. The measured velocity widths $\left(\Delta v_{\text {FWHM }} \sim 600-850 \mathrm{~km} \mathrm{~s}^{-1}\right.$ for the NLR lines and $\Delta v_{\text {FWHM }} \sim$ $5500 \mathrm{~km} \mathrm{~s}^{-1}$ for the BLR line) are consistent with typical values for NLR and BLR emission lines seen in type 1 AGNs $\left(426 \pm 251 \mathrm{~km} \mathrm{~s}^{-1}\right.$ and $4420 \pm 3210 \mathrm{~km} \mathrm{~s}^{-1}$ for the NLR and BLR lines; Ho \& Kim 2009). Note that the systematic errors given for the emission-line widths were estimated by applying some fitting functions for emission lines (such as Gaussian, Lorentzian, and so on) and examining the standard deviation of the width. Here the statistical errors in the velocity width are smaller than the systematic errors, given the achieved signal-to-noise ratio.

\section{Discussion}

\subsection{The chemical properties of the narrow line region}

One of the aims of this study is to estimate the metallicity of the host galaxy of the N-loud quasar SDSS J1707+6443. More specifically, we wish to test whether this quasar has extremely metal-rich gas clouds, as expected from its strong broad nitrogen emission lines. Although strong broad nitrogen lines are usually interpreted as indications of high $Z_{\mathrm{BLR}}$ (for both permitted lines and semi-forbidden lines; see, e.g., Shields 1976; Hamann \& Ferland 1992), Jiang et al. (2008) pointed out that N-loud quasars might simply have unusually high nitrogen relative abundances. This is because the emission-line spectrum of the $\mathrm{N}$-loud quasars is similar to that of common type 1 quasars. In particular, many N-loud quasars show no anomalous behavior in other broad emission-line flux ratios, not involving nitrogen, but are sensitive to $Z_{\mathrm{BLR}}$, such as ( $\mathrm{Si}$ IV $+\mathrm{O}$ IV])/C IV (Nagao et al. 2006b; Juarez et al. 2009; Simon \& Hamann 2010). However, the nuclear BLR involves only a very small fraction of the gas content in the galaxy and, therefore, may well not be representative of the metallicity in the host galaxy. As a consequence, it may be more instructive to investigate the metallicity in the $\operatorname{NLR}\left(Z_{\mathrm{NLR}}\right)$ through the rest-frame optical spectrum to investigate whether the high metallicities inferred for the BLR are confirmed on the larger scales traced by the NLR.

We focus first on the metallicity dependence of the line emissivity on the physical conditions typical of the NLR. The emissivity of collisionally excited emission lines strongly depends on the gas temperature. The equilibrium temperature of ionized gas clouds depends on gas metallicity, because metal emission lines are the main coolants of these clouds. Therefore, collisionally excited emission lines from the NLR become fainter for increasing $Z_{\text {NLR }}$ (e.g., Nagao et al. 2006a; Matsuoka et al. 2009). To show this effect more explicitly, we computed simple photoionization model calculations with Cloudy, version 08.00 (Ferland et al. 1998; Ferland 2006). We assumed ionization-bounded plane-parallel gas clouds with a constant density, photoionized by the typical spectral energy distribution for quasars (Mathews \& Ferland 1987; Ferland 2006). We examined the metallicity dependence of the equivalent width (EW) of the [O III] emission with the relative elemental abundance ratios fixed to the solar values except for nitrogen. We assumed that the nitrogen relative abundance $(\mathrm{N} / \mathrm{H})$ scales with the square of the metallicity. In Fig. 6, we show photoionization model results for gas densities of $n_{\mathrm{H}}=10^{1} \mathrm{~cm}^{-3}$ and $10^{4} \mathrm{~cm}^{-3}$, and ionization parameters of $U=10^{-3.5}$ and $10^{-1.5}$. The emissivity curves in this figure are normalized by their peak to highlight their dependence on the gas metallicity. Clearly, the $E W([\mathrm{O}$ III]) decreases rapidly as the metallicity increases, and the resulting $E W([\mathrm{O} \mathrm{III}])$ is quite small at $Z_{\mathrm{NLR}}>5 Z_{\odot}$. Note that we cannot derive accurate values of $Z_{\mathrm{NLR}}$ based only on [O III], since detailed metallicity studies require multiple emission lines (e.g., Storchi-Bergmann et al. 1998; Nagao et al. 2002; Iwamuro et al. 2003; Groves et al. 2006; Nagao et al. 2006a; Matsuoka et al. 2009). However, the aim of this paper is only to assess whether the NLR in SDSS J1707+6443 has a high metallicity, not to derive its accurate value. These results suggest that we can distinguish whether or not $\mathrm{N}$-loud quasars have a relatively high $Z_{\mathrm{NLR}}$ with respect to other type 1 quasars by comparing the $E W([\mathrm{O} \mathrm{III}])$ distributions of the two populations. 


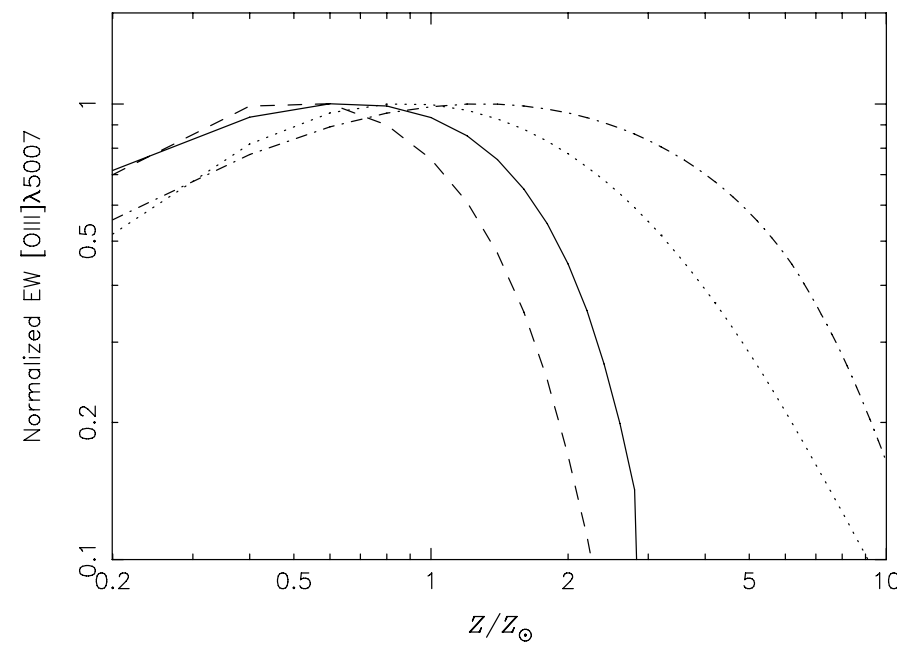

Fig. 6. Predicted equivalent width of $[\mathrm{O}$ III $] \lambda 5007$, as a function of $Z_{\mathrm{NLR}}$. Solid, dashed, dot-dashed, and dotted lines denote the models with $\left(\log n_{\mathrm{H}}, \log U\right)=(1,-1.5),(1,-3.5),(4,-1.5)$, and $(4,-3.5)$, respectively. The EW predictions are normalized by their peak values.

In Fig. 7, we show the frequency distribution of $E W_{\text {rest }}([\mathrm{O}$ III $])$ of type 1 quasars at $1<z<4$, which are compiled from the works of McIntosh et al. (1999), Netzer et al. (2004), Marziani et al. (2009), and Greene et al. (2010) (shown with a solid histogram in Fig. 7). In these four papers, there are 86 [O III]-detected quasars, whose average and median $\mathrm{EW}([\mathrm{O} \text { III }])_{\text {rest }}$ values are $16.5 \AA$ and $13.0 \AA$ with the standard deviation of $14.6 \AA$ (the minimum and maximum values are $0.4 \AA$ and $73.0 \AA$, respectively). Since the $E W_{\text {rest }}([\mathrm{O}$ III] $])$ of SDSS J1707+6443 is $17.4 \AA$ (Table 2), the [O III] emission of SDSS J1707+6443 is not weaker than that of typical type 1 quasars. In Fig. 7 we also show the frequency distribution of $E W_{\text {rest }}([\mathrm{O}$ III]) of SDSS quasars at $0<z<1$ (shown with filled circles with error bars in Fig. 7). This is the distribution by Risaliti et al. (2011), who used the spectral measurements of SDSS DR5 quasars (Schneider et al. 2007) performed by Shen et al. (2008). The frequency distribution of $E W_{\text {rest }}([\mathrm{O}$ III $]$ ) of the $0<z<1$ SDSS sample (Risaliti et al. 2011; filled circles in Fig. 7) is not significantly different from that of the higher- $z(1<z<4)$ sample (solid histogram in Fig. 7). The $E W_{\text {rest }}([\mathrm{O}$ III] $)$ value of SDSS J1707+6443 is higher than the peak value of these distributions. Therefore we conclude that the $E W_{\text {rest }}([\mathrm{O} \mathrm{III}])$ of SDSS J1707+6443 is not significantly smaller than that of typical type 1 quasars in both low- $z$ and high- $z$ samples. Note that the actual relative [O III] $\lambda 5007$ strength of SDSS J1707+6443 with respect to the whole parent sample of type 1 quasars could be higher, because only [O III]detected quasars (i.e., relatively strong [O III] emitters) are selectively shown in Fig. 7.

The relatively large $E W\left([\mathrm{O} \text { III] })_{\text {rest }}\right.$ of SDSS $\mathrm{J} 1707+6443$ with respect to the global population of AGNs (Fig. 7) suggests that the NLR in SDSS J1707+6443 is not characterized by a very high $Z_{\mathrm{NLR}}$, when the photoionization model results shown in Fig. 6 are taken into account. If a positive correlation between $Z_{\mathrm{NLR}}$ and $Z_{\mathrm{BLR}}$ is assumed, a lack of very high metallicity clouds in the NLR seems to be inconsistent with the strong broad nitrogen lines seen in the rest-frame UV spectrum of this object. Obviously these are two possible scenarios to explain this inconsistency: (1) the strong UV broad nitrogen lines are caused by a very high relative abundance of nitrogen in the BLR (with respect to non-N-loud quasars) and not by a BLR

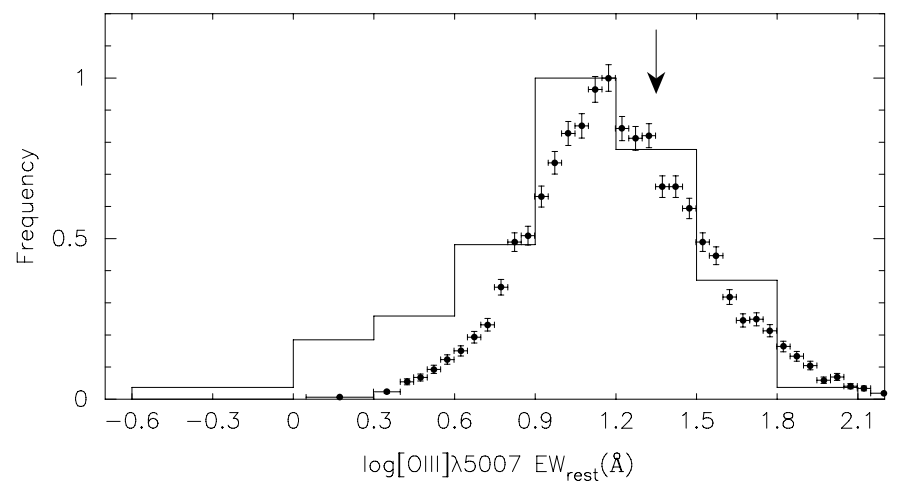

Fig. 7. Solid-line histogram denotes the compiled data of high- $z$ type 1 quasars from the literature (McIntosh et al. 1999; Netzer et al. 2004; Marziani et al. 2009; Greene et al. 2010). Filled circles with error bars denote the data of SDSS type 1 quasars at $0<z<1$ (Risaliti et al. 2011). The frequency distributions of the two datasets are normalized by the number of objects in the bin at the peak of their frequency distributions. The arrow denotes the $E W_{\text {rest }}\left(\left[\mathrm{O}_{\mathrm{III}}\right]\right)$ value of SDSS J1707+6443 measured in our work.

metallicity extremely higher than non-N-loud quasars, or (2) the BLR metallicity is significantly higher than the NLR metallicity, which better represents the metallicity of the host galaxy. The relation between $Z_{\mathrm{BLR}}$ and $Z_{\mathrm{NLR}}$ is not yet well understood observationally, although Fu \& Stockton $(2007,2008,2009)$ reported that these two quantities are probably related with each other, at least in low-z $(z<0.5)$ quasars. Whatever case applies, (1) or (2), the strong broad nitrogen lines of SDSS J1707+6443 are not consistent with the possibility that the host galaxy of this quasar is characterized by a very high metallicity. This implies that broad UV nitrogen lines of quasars are not (at least in some cases) a good tool to explore the chemical evolution of quasar host galaxies. Here we note that the spatial scale of NLRs is far larger than that of BLRs but does not necessarily coincide with the spatial scale of their host galaxies; we show for SDSS J1707+6443, however, that the NLR spatial scale corresponds to the host-galaxy scale ( $\sim \mathrm{kpc}$ scale; see Sect. 4.3$)$. Since quasar spectra are frequently used to investigate the chemical evolution at high redshifts, it will be crucial to examine whether the broad UV lines of quasars are good (or bad) tracers of the metallicity through more detailed studies for larger samples of AGNs (see, e.g., Matsuoka et al. 2011).

\subsection{Black hole mass and the Eddington ratio}

The results in the previous section suggest that the strong nitrogen emission in the BLR of SDSS J1707+6443 (or, possibly, $\mathrm{N}$-loud quasars in general) is not indicative of high metallicity in the NLR and hence in the host galaxy. It is important, however, to verify whether the properties of the broad nitrogen lines of SDSS J1707+6443 do follow the trends of the global population of high- $z$ type 1 quasars. Recently Matsuoka et al. (2011) found that a high relative nitrogen abundance is seen in BLRs of high- $L_{\text {bol }} / L_{\text {Edd }}$ quasars. To examine whether SDSS J1707+6443 is consistent with the result of Matsuoka et al. (2011), we estimated $M_{\mathrm{BH}}$ and $L_{\mathrm{bol}} / L_{\mathrm{Edd}}$ based on the velocity widths of C IV in the SDSS spectrum and $\mathrm{H} \beta$ in our MOIRCS spectrum. Note that it is debatable whether $\mathrm{C}$ IV-based or $\mathrm{H} \beta$-based estimations are more accurate (see, e.g., Netzer et al. 2004; Peterson et al. 2004; Denney et al. 2009). Therefore we used both emission lines to minimize possible systematic errors in the estimation of those parameters. 
We derived $M_{\mathrm{BH}}$ for SDSS J1707+6443 by adopting the calibrations given by Vestergaard \& Peterson (2006),

$\log \frac{M_{\mathrm{BH}}}{M_{\odot}}=6.66+0.53 \log \left(\frac{\lambda L_{\lambda}(1350 \AA)}{10^{44} \mathrm{erg} \mathrm{s}^{-1}}\right)+2 \log \left(\frac{F W H M}{1000 \mathrm{~km} \mathrm{~s}^{-1}}\right)$

for C IV, and

$\log \frac{M_{\mathrm{BH}}}{M_{\odot}}=6.91+0.50 \log \left(\frac{\lambda L_{\lambda}(5100 \AA)}{10^{44} \mathrm{erg} \mathrm{s}^{-1}}\right)+2 \log \left(\frac{F W H M}{1000 \mathrm{~km} \mathrm{~s}^{-1}}\right)$

for $\mathrm{H} \beta$, where $F W M H_{\mathrm{CIV}}$ and $F W H M_{\mathrm{H} \beta}$ are the velocity width of the $\mathrm{C}$ IV and $\mathrm{H} \beta$ emission in full-width at half maximum, respectively. For estimating the Eddington ratio $\left(L_{\text {bol }} / L_{\text {Edd }}\right)$, we adopted a bolometric correction of $L_{\mathrm{bol}}=9.26 \lambda L_{\lambda} 5100$ (Shen et al. 2008). The derived values are $\log \left(M_{\mathrm{BH}} / M_{\odot}\right)=8.98$ and $\log \left(L_{\mathrm{bol}} / L_{\mathrm{Edd}}\right)=-0.53$ when using $\mathrm{C}$ IV , and $\log \left(M_{\mathrm{BH}} / M_{\odot}\right)=$ 9.73 and $\log \left(L_{\text {bol }} / L_{\text {Edd }}\right)=-0.21$ when using $\mathrm{H} \beta$, respectively. Accordingly we adopted their means, $\log \left(M_{\mathrm{BH}} / M_{\odot}\right)=9.50$ and $\log \left(L_{\mathrm{bol}} / L_{\mathrm{Edd}}\right)=-0.34$. The derived Eddington ratio is relatively high (regardless of the adopted emission line for deriving $M_{\mathrm{BH}}$ ), with respect to the frequency distribution of the Eddington ratio of SDSS type 1 quasars at similar redshifts $(-0.48 \pm 0.41$ for 3144 quasars at $3.0<z<3.3$; Shen et al. 2011).

Our results is consistent with the finding of Matsuoka et al. (2011) that quasars with a high accretion rate are not necessarily characterized by high metallicities, but are characterized by high nitrogen abundances (resulting in stronger nitrogen lines). In the scenario proposed by Matsuoka et al. (2011), the high black hole accretion is delayed by a few $100 \mathrm{Myr}$, relative to the main episode of star formation, when intermediate-mass stars have evolved and have enriched the ISM with nitrogen.

\subsection{Emission-line diagnostics in the narrow line region}

By combining the emission-line fluxes of $[\mathrm{O}$ III] $\lambda 5007$ and $[\mathrm{Ne}$ III] $] 33869$ and the upper limit on the flux of $[\mathrm{O}$ II] $] \lambda 3727$, it is possible to investigate the properties of NLR gas clouds. This analysis enables us to study the physical properties of gas clouds in the host galaxy, because the NLR is extended on galactic scales in contrast to the BLR. Moreover, since the redshift of SDSS J1707+6443 $(z \sim 3)$ corresponds to the peak of the global quasar activity (e.g., Richards et al. 2006), the gas properties of the quasar host galaxy are interesting to explore the interplay between AGNs and their host galaxies (i.e., the galaxy-SMBH coevolution). In this context, host galaxies of the N-loud quasars are particularly interesting since they may be in a special evolutionary stage, as mentioned in Sect. 4.2. Note that NLR emission lines other than $[\mathrm{O}$ III] $\lambda 5007$ have only rarely been observed in luminous high-z type 1 quasars, which prevents a detailed investigation of NLR gas properties similar to our MOIRCS spectrum.

In Fig. 8, we place SDSS J1707+6443 on the diagram comparing the distributions of the emission-line flux ratios of [O II] $\lambda 3727 /[\mathrm{O}$ III] $] \lambda 5007$ and [Ne III] $\lambda 3869 /[\mathrm{O}$ III] $] \lambda 5007$. For comparison, we also plot the data of the SDSS DR7 type 1 quasar sample (Shen et al. 2011). We checked their SDSS archival spectra and selected quasars whose [O II] $] 3727$, $[\mathrm{Ne}$ III] $] 3869$, and [O III] $\lambda 5007$ are significantly detected (i.e., $S / N>10)$. As a consequence we plot the emission-line flux ratio of 25 SDSS DR7 type 1 quasars, whose redshift range is $0.37<z<0.80$ with the median redshift of 0.65 .

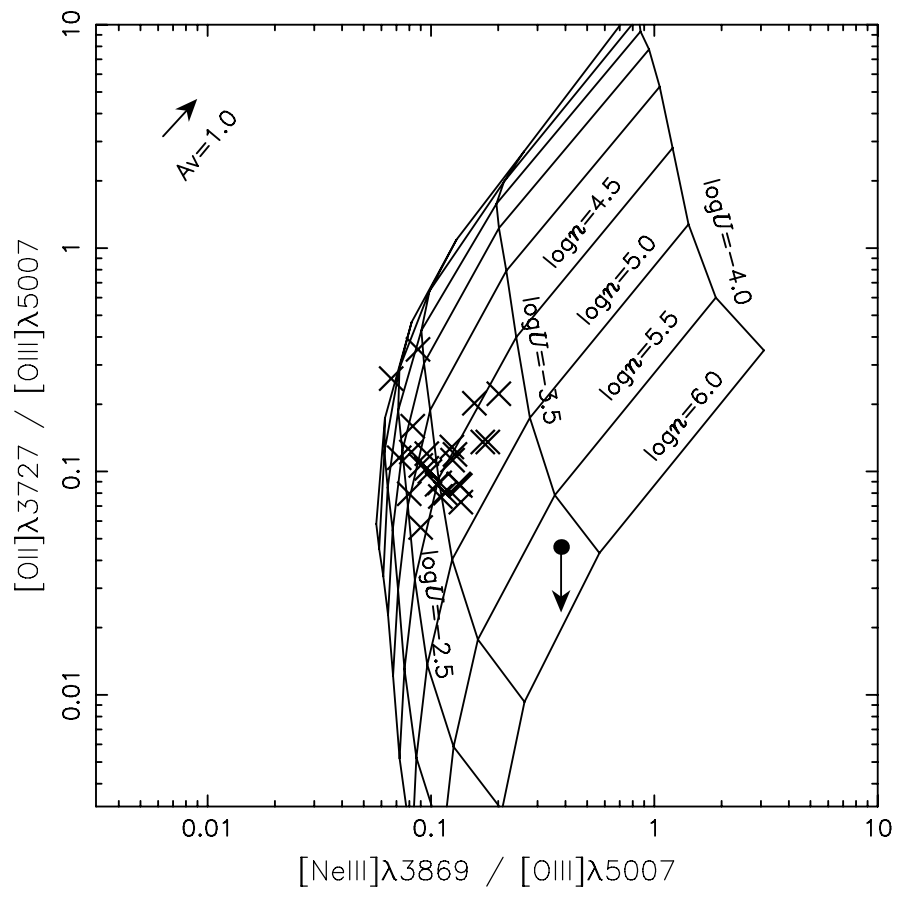

Fig. 8. Emission-line flux ratios of $[\mathrm{O}$ II $] \lambda 3727 /[\mathrm{O}$ III] $] \lambda 5007$ versus $[\mathrm{Ne}$ III] $] \lambda 3869 /[\mathrm{O}$ III] $] \lambda 5007$. The filled circle with an arrow denotes the data of SDSS J1707+6443 (where the $3 \sigma$ upper limit is adopted for the [O II] flux), and cross symbols denote the data of the SDSS low- $z$ $(z \sim 0.7)$ type 1 quasars. The arrow at the upper-left corner in the panel is the reddening vector for observed data in the case of $A_{V}=1.0 \mathrm{mag}$, adopting the extinction curve of Cardelli et al. (1989). The grids are predicted emission-line flux ratios from Cloudy model runs, adopting $Z_{\mathrm{NLR}}=3 Z_{\odot}$ and varying the hydrogen density and the ionization parameter.

Note that the average luminosity $\lambda L_{\lambda}(5100)$ for the 25 SDSS type 1 quasars is $10^{45.77} \mathrm{erg} \mathrm{s}^{-1}$, that is $\sim 1$ dex lower than the corresponding value for SDSS J1707+6443 $\left(10^{46.66} \mathrm{erg} \mathrm{s}^{-1}\right)$. As shown in Fig. 8, these 25 SDSS type 1 quasars show completely different flux ratios from SDSS J1707+6443; i.e., SDSS J1707+6443 shows a higher [Ne III] $\lambda 3869 /[\mathrm{O}$ III] $] 25007$ ratio and a lower [O II] $\lambda 3727 /[\mathrm{O}$ III] $\lambda 5007$ ratio than SDSS type 1 quasars. These differences can be interpreted qualitatively if the NLR in SDSS J1707+6443 is characterized by a much higher average density than the NLRs in SDSS type 1 quasars at $z<1$. This is because the [O $\mathrm{II}] \lambda 3727$ emission and partly [O III] $\lambda 5007$ emission are suppressed by the collisional de-excitation process in high-density clouds with $n_{\mathrm{H}} \sim 10^{6} \mathrm{~cm}^{-3}$ or higher, by taking the critical densities of these emission lines $\left(n_{\mathrm{cr}}\left(\left[\mathrm{O}_{\mathrm{II}}\right]\right)=(3.3-14) \times 10^{3} \mathrm{~cm}^{-3}, n_{\mathrm{cr}}\left(\left[\mathrm{O}_{\mathrm{III}}\right]\right)=7.0 \times 10^{5} \mathrm{~cm}^{-3}\right.$, and $\left.n_{\mathrm{cr}}([\mathrm{Ne} \mathrm{III}])=9.7 \times 10^{6} \mathrm{~cm}^{-3}\right)$ into account.

To investigate the differences in the NLR emission-line flux ratios between SDSS J1707+6443 and lower- $z$ type 1 quasars more quantitatively, we have performed Cloudy model runs for the parameter ranges of $n_{\mathrm{H}}=10^{2}-10^{6} \mathrm{~cm}^{-3}$ and $U=10^{-4.0}-10^{-1.5}$. Here we adopted a metallicity of $Z_{\mathrm{NLR}}=3 Z_{\odot}$, which is the typical value for NLR clouds (e.g., Groves et al. 2006), because we concluded in Sect. 4.1 that SDSS J1707+6443 is not characterized by very high $Z_{\mathrm{NLR}}$. The other adopted parameters such as the input SED are the same as those described in Sect. 4.1. The results of the model runs are overlaid in Fig. 8. The NLRs in the SDSS type 1 quasars are characterized by $n_{\mathrm{H}} \sim 10^{4.5} \mathrm{~cm}^{-3}$ and $U \sim 10^{-3.5}-10^{-2.5}$, consistent with the parameter ranges inferred by previous studies 
(e.g., Nagao et al. 2001b). On the other hand, the NLR in SDSS J1707+6443 is characterized by a higher density, $n_{\mathrm{H}} \sim$ $10^{6} \mathrm{~cm}^{-3}$ or more, although the ionization parameter is similar to that of type 1 SDSS quasars. These results are not sensitive to the adopted metallicity.

There are two possible scenarios to explain the distinct physical properties of SDSS J1707+6443. One possibility is considering a strong contribution from the inner wall of the dusty torus. Because the clouds located at the inner side of dusty tori are characterized by a high density and photoionized by the strong central continuum radiation, emission lines with high critical densities are radiated from these clouds (e.g., Murayama \& Taniguchi 1998b; Nagao et al. 2000, 2001a). If these high-density clouds at the inner dusty torus show strong NLR emission, its emission dominates the whole NLR emission and then low- $n_{\mathrm{cr}}$ emission lines become relatively weak, explaining the observed emission-line flux ratios of SDSS J1707+6443 (see also Murayama \& Taniguchi 1998a; Nagao et al. 2001c). Another possibility is the existence of high-density clouds distributed on galactic scales (i.e., $\sim \mathrm{kpc}$ ). Such a situation has been proposed for high- $z$ and/or high-luminosity quasars (Netzer et al. 2004; see also Ho 2005), possibly related to violent starformation activity in the host galaxy. Note that the latter scenario is not ruled out by the non-detection of the $\left[\mathrm{O}_{\mathrm{II}}\right] \lambda 3727$ emission, which is used as an indicator of the star formation rate (e.g., Gallagher et al. 1989; Kennicutt 1998; Ideue et al. 2009). This is because the [O II] $\lambda 3727$ emission is significantly suppressed in high-density $\mathrm{H}$ II regions when the density is higher than $10^{4} \mathrm{~cm}^{-3}$, even when the star formation activity is very vigorous.

To distinguish between the above two scenarios, we estimated the typical distance of the ionized clouds from the nucleus in SDSS J1707+6443 by combining the estimates of $n_{\mathrm{H}}$ and $U$ obtained in the previous section. Since we already estimated $L_{\text {bol }}$ of SDSS J1707+6443 in Sect. 4.1, the number of the ionizing photon can be estimated for a given spectral energy distribution (SED). Here we assumed the same SED as in the above photoionization model calculations (Mathews \& Ferland 1987) and adopted the parameters of $n_{\mathrm{H}}=10^{6} \mathrm{~cm}^{-3}$ and $U=10^{-3.5}$. Accordingly, we obtained the result that the typical distance of the clouds is $\sim 1.5 \mathrm{kpc}$, which is consistent with the latter scenario that considers high-density clouds in the host galaxy. Note that a smaller radius would have been derived by assuming a higher density, e.g., $\sim 15$ pc for $n_{\mathrm{H}} \sim 10^{8} \mathrm{~cm}^{-3}$. However, this is not plausible, because [O III] $\lambda 5007$ emission would be significantly suppressed at such high-densities due to the collisional de-excitation. We thus conclude that there are high-density clouds at the kpc scale in the host galaxy of SDSS J1707+6443, which dominate the emission of the detected NLR lines. The torus scenario is disfavored also in terms of the observed NLR velocity width. By assuming that the SMBH is the dominant source of the gravitational potential field in the nucleus of SDSS J1707+6443, the expected velocity width of [O III] $\lambda 5007$ is $\sim 1300 \mathrm{~km} \mathrm{~s}^{-1}$ if the most of this emission arises at $\sim 15 \mathrm{pc}$ from the SMBH with $M_{\mathrm{BH}}=10^{9.78} M_{\odot}$ (see Sect. 4.2). This expected velocity width is apparently larger than the observed width $\left(617 \mathrm{~km} \mathrm{~s}^{-1}\right.$; Table 2), suggesting that most of the [O III $] \lambda 5007$ emission in this object arises at larger spatial scales, which probably correspond to the scale of its host galaxy.

Here we briefly discuss the implication of the extended $(\sim \mathrm{kpc})$ dense $\left(\sim 10^{6} \mathrm{~cm}^{-3}\right)$ gas clouds in SDSS J1707+6443 inferred from emission-line diagnostics. It is well known that these dense clouds in galaxies are closely related to the star-forming activity. Plume et al. (1997) reported their observations of the carbon monosulphide (CS) molecule for $>100$ Galactic $\mathrm{H}$ II regions and showed that their typical density is $n \sim 10^{6} \mathrm{~cm}^{-3}$ (but less than $2 \times 10^{7} \mathrm{~cm}^{-3}$ ). Such a high density for clouds in $\mathrm{H}$ II regions has been inferred also from some other molecularline radio observations (e.g., Hofner et al. 1996; Bergin et al. 1996; Lada et al. 1997). Although such a high density is not the "typical" density for $\mathrm{H}$ II region clouds, the star formation efficiency of the denser clouds is actually much higher than less dense clouds. This is suggested by, e.g., a clear positive correlation between the $L_{\mathrm{HCN}} / L_{\mathrm{CO}}$ ratio (i.e., the dense-gas fraction) and the $L_{\mathrm{IR}} / L_{\mathrm{CO}}$ ratio (i.e., the star-formation efficiency) that is seen in H II region clouds (e.g., Gao \& Solomon 2004). Therefore high-density clouds generally dominate the star formation, when they exist (see also, e.g., Wang et al. 2011). A similar situation is also seen in high- $z$ galaxies (e.g., Gao et al. 2007; Riechers et al. 2007, 2010, 2011) but with a significant difference compared to low- $z$ galaxies; that is, the spatial extension of vigorous star-forming regions. The spatial scale of starforming regions in high- $z$ actively star-forming galaxies (such as sub-millimeter galaxies) extends up to $\sim \mathrm{kpc}$ scales or more (e.g., Chapman et al. 2004; Walter et al. 2009), which is different from low- $z$ actively star-forming galaxies such as ultra-luminous infrared galaxies (e.g., Downes \& Solomon 1998; see also, e.g., Iono et al. 2009). These pictures well match with our results in the sense that dense gas clouds $\left(\sim 10^{6} \mathrm{~cm}^{-3}\right)$ are distributed at the $\sim \mathrm{kpc}$ scale in a galaxy at $z \sim 3.2$. Therefore, given these considerations, we speculate that there is ongoing vigorous starforming activity in SDSS J1707+6443.

\section{Summary}

To assess the physical and chemical properties of the host galaxies of N-loud quasars, we analyzed the MOIRC near-infrared spectrum of SDSS J1707+6443, at $z=3.16$, obtaining the following results:

- this N-loud quasar shows strong [O III] $\lambda 5007$ emission. Since photoionization models predict weak $[\mathrm{O}$ III $] \lambda 5007$ emission when the NLR metallicity is very high $\left(>5 Z_{\odot}\right)$, the detected strong [O III] $\lambda 5007$ emission suggests that the NLR in this object is not characterized by very high metallicities;

- the UV nitrogen lines from BLRs are not ideal tools to discuss the chemical evolution of quasar host galaxies, because whatever the origin of strong broad nitrogen emission (high $Z_{\mathrm{BLR}}$, or high relative abundance of nitrogen with an ordinary metallicity), $Z_{\mathrm{BLR}}$ are likely unrelated with the metallicities of the host galaxies;

- the Eddington ratio of SDSS J1707+6443 is moderately high $\left(L_{\mathrm{bol}} / L_{\mathrm{Edd}}=10^{-0.26}\right)$, as derived from single-epoch $M_{\mathrm{BH}}$ estimates using both $\mathrm{C}$ IV and $\mathrm{H} \beta$ emission lines. This is consistent with the discovery by Matsuoka et al. (2011) that a high relative nitrogen abundance is associated to quasars with high Eddington ratios;

- the flux ratio of $\left[\begin{array}{ll}\mathrm{O} & \mathrm{II}\end{array}\right] \lambda 3727 /\left[\begin{array}{ll}\mathrm{O} & \mathrm{III}\end{array}\right] \lambda 5007$ in SDSS J1707+6443 is significantly lower than that in lower- $z$ type 1 quasars, indicating that NLR clouds in SDSS $\mathrm{J} 1707+6443$ are characterized by much higher densities than those in other type 1 quasars;

- photoionization models suggest that those high-density clouds (with $n_{\mathrm{H}} \sim 10^{6} \mathrm{~cm}^{-3}$ ) are located at the kpc scales in the host galaxy of SDSS J1707+6443, and we speculate that this might be related to the vigorous star-formation activity in the host galaxy. 
These results possibly reveal a strong connection between the high AGN activity (characterized by a high Eddington ratio) and the vigorous star formation activity suggested by the kpc-scale distribution of dense gas cloud in a N-loud quasar, SDSS J1707+6443. Currently it is not clear whether such a connection is seen also in other N-loud quasars generally or not, given the paucity of detailed near-infrared spectroscopic studies for N-loud quasars. Therefore it is highly interesting to examine whether such a situation is common in other N-loud quasars through more near-infrared spectroscopic observations for a large sample of $\mathrm{N}$-loud quasars.

Acknowledgements. We thank the Subaru Telescope staff for supporting our MOIRCS observation. We also thank the anonymous referee, Takayuki Saitoh, and Bunyo Hatsukade, for their useful comments. Funding for the creation and distribution of the SDSS Archive has been provided by the Alfred P. Sloan Foundation, the Participating Institutions, the National Aeronautics and Space Administration, the National Science Foundation, the US Department of Energy, the Japanese Monbukagakusyo, and the Max Planck Society. The SDSS web site is http: //www.sdss.org/. We thank Gary Ferland for providing his photoionization code Cloudy to the public. N.A. is supported in part by a grant from the Hayakawa Satio Fund awarded by the Astronomical Society of Japan. T.N. is financially supported by JSPS (grant no. 23654068), the Kurata Memorial Hitachi Science and Technology Foundation, the Itoh Science Foundation, Ehime University (the Research Promotion Award), and Kyoto University (the Hakubi Project grant). K.M. acknowledges financial support from JSPS through JSPS Research Fellowships for Young Scientists. Y.T. is financially supported by JSPS (grant no. 23244031).

\section{References}

Baldwin, J. A., Hamann, F., Korista, K. T., et al. 2003, ApJ, 583, 649 Ballero, S. K., Matteucci, F., Ciotti, L., Calura, F., \& Padovani, P. 2008, A\&A, 478,335

Bentz, M. C., \& Osmer, P. S. 2004, AJ, 127, 576

Bentz, M. C., Hall, P. B., \& Osmer, P. S. 2004, AJ, 128, 561

Bergin, E. A., Snell, R. L., \& Goldsmith, P. F. 1996, ApJ, 460, 343

Cardelli, J. A., Clayton, G. C., \& Mathis, J. S. 1989, ApJ, 345, 245

Chapman, S. C., Smail, I., Windhorst, R., Muxlow, T., \& Ivison, R. J. 2004, ApJ, 611,732

Denney, K. D., Watson, L. C., Peterson, B. M., et al. 2009, ApJ, 702, 1353

Downes, D., \& Solomon, P. M. 1998, ApJ, 507, 615

Ferland, G. J. 2006, Hazy, A Brief Introduction to Cloudy 06.02

Ferland, G. J., Korista, K. T., Verner, D. A., et al. 1998, PASP, 110, 761

Ferrarese, L., \& Merritt, D. 2000, ApJ, 539, L9

Fu, H., \& Stockton, A. 2007, ApJ, 664, L75

Fu, H., \& Stockton, A. 2008, ApJ, 677, 79

Fu, H., \& Stockton, A. 2009, ApJ, 696, 1693

Gallagher, J. S., Hunter, D. A., \& Bushouse, H. 1989, AJ, 97, 700

Gao, Y., Carilli, C. L., Solomon, P. M., \& Vanden Bout, P. A. 2007, ApJ, 660, L93

Gao, Y., \& Solomon, P. M. 2004, ApJ, 606, 271

Gebhardt, K., Bender, R., Bower, G., et al. 2000, ApJ, 539, L13

Greene, J. E., Peng, C. Y., \& Ludwig, R. R. 2010, ApJ, 709, 937

Groves, B. A., Heckman, T. M., \& Kauffmann, G. 2006, MNRAS, 371, 1559

Hamann, F., \& Ferland, G. 1992, ApJ, 391, L53
Hamann, F., \& Ferland, G. 1993, ApJ, 418, 11

Ho, L. C. 2005, ApJ, 629, 680

Ho, L. C., \& Kim, M. 2009, ApJS, 184, 398

Hofner, P., Kurtz, S., Churchwell, E., Walmsley, C. M., \& Cesaroni, R. 1996, ApJ, 460, 359

Ichikawa, T., Suzuki, R., Tokoku, C., et al. 2006, in SPIE Conf., 6269, 626916

Ideue, Y., Nagao, T., Taniguchi, Y., et al. 2009, ApJ, 700, 971

Iono, D., Wilson, C. D., Yun, M. S., et al. 2009, ApJ, 695, 1537

Iwamuro, F., Motohara, K., Maihara, T., et al. 2003, ApJ, 598, 178

Jiang, L., Fan, X., Vestergaard, M., et al. 2007, AJ, 134, 1150

Jiang, L., Fan, X., \& Vestergaard, M. 2008, ApJ, 679, 962

Juarez, Y., Maiolino, R., Mujica, R., et al. 2009, A\&A, 494, L25

Kennicutt, Jr., R. C. 1998, ARA\&A, 36, 189

Kriss, G. 1994, Astronomical Data Analysis Software and Systems, 3, 437

Lada, E. A., Evans, II, N. J., \& Falgarone, E. 1997, ApJ, 488, 286

Marconi, A., \& Hunt, L. K. 2003, ApJ, 589, L21

Marziani, P., Sulentic, J. W., Stirpe, G. M., Zamfir, S., \& Calvani, M. 2009, A\&A, 495, 83

Mathews, W. G., \& Ferland, G. J. 1987, ApJ, 323, 456

Matsuoka, K., Nagao, T., Maiolino, R., Marconi, A., \& Taniguchi, Y. 2009, A\&A, 503, 721

Matsuoka, K., Nagao, T., Marconi, A., Maiolino, R., \& Taniguchi, Y. 2011, A\&A, 527, A100

McIntosh, D. H., Rieke, M. J., Rix, H., Foltz, C. B., \& Weymann, R. J. 1999, ApJ, 514, 40

Mortlock, D. J., Warren, S. J., Venemans, B. P., et al. 2011, Nature, 474, 616

Murayama, T., \& Taniguchi, Y. 1998a, ApJ, 503, L115

Murayama, T., \& Taniguchi, Y. 1998b, ApJ, 497, L9

Nagao, T., Taniguchi, Y., \& Murayama, T. 2000, AJ, 119, 2605

Nagao, T., Murayama, T., \& Taniguchi, Y. 2001a, PASJ, 53, 629

Nagao, T., Murayama, T., \& Taniguchi, Y. 2001b, ApJ, 546, 744

Nagao, T., Murayama, T., \& Taniguchi, Y. 2001c, ApJ, 549, 155

Nagao, T., Murayama, T., Shioya, Y., \& Taniguchi, Y. 2002, ApJ, 575, 721

Nagao, T., Maiolino, R., \& Marconi, A. 2006a, A\&A, 447, 863

Nagao, T., Marconi, A., \& Maiolino, R. 2006b, A\&A, 447, 157

Netzer, H., Shemmer, O., Maiolino, R., et al. 2004, ApJ, 614, 558

Peterson, B. M., Ferrarese, L., Gilbert, K. M., et al. 2004, ApJ, 613, 682

Plume, R., Jaffe, D. T., Evans, II, N. J., Martin-Pintado, J., \& Gomez-Gonzalez, J. 1997, ApJ, 476, 730

Richards, G. T., Strauss, M. A., Fan, X., et al. 2006, AJ, 131, 2766

Riechers, D. A., Walter, F., Carilli, C. L., \& Bertoldi, F. 2007, ApJ, 671, L13

Riechers, D. A., Weiß, A., Walter, F., \& Wagg, J. 2010, ApJ, 725, 1032

Riechers, D. A., Walter, F., Carilli, C. L., et al. 2011, ApJ, 726, 50

Risaliti, G., Salvati, M., \& Marconi, A. 2011, MNRAS, 411, 2223

Schneider, D. P., Hall, P. B., Richards, G. T., et al. 2007, AJ, 134, 102

Shen, Y., Greene, J. E., Strauss, M. A., Richards, G. T., \& Schneider, D. P. 2008, ApJ, 680, 169

Shen, Y., Richards, G. T., Strauss, M. A., et al. 2011, ApJS, 194, 45

Shields, G. A. 1976, ApJ, 204, 330

Simon, L. E., \& Hamann, F. 2010, MNRAS, 407, 1826

Storchi-Bergmann, T., Schmitt, H. R., Calzetti, D., \& Kinney, A. L. 1998, AJ, 115,909

Suzuki, R., Tokoku, C., Ichikawa, T., et al. 2008, PASJ, 60, 1347

Vanden Berk, D. E., Richards, G. T., Bauer, A., et al. 2001, AJ, 122, 549

Vestergaard, M., \& Peterson, B. M. 2006, ApJ, 641, 689

Walter, F., Riechers, D., Cox, P., et al. 2009, Nature, 457, 699

Wang, J., Zhang, Z., \& Shi, Y. 2011, MNRAS, 416, L21

Warner, C., Hamann, F., \& Dietrich, M. 2004, ApJ, 608, 136

York, D. G., Adelman, J., Anderson, Jr., J. E., et al. 2000, AJ, 120, 1579 